OPEN ACCESS

Edited by:

Yuping Wang,

Xuanwu Hospital, Capital Medical

University, China

Reviewed by:

Marino M. Bianchin,

Universidade Federal do Rio Grande

do Sul (UFRGS), Brazi

Jose F. Tellez-Zenteno,

University of Saskatchewan, Canada

*Correspondence:

Da E. Jung

j978005@naver.com

Heung D. Kim

hdkimmd@yuhs.ac

tThese authors have contributed equally to this work.

Specialty section:

This article was submitted to

Epilepsy,

a section of the journal

Frontiers in Neurology

Received: 27 December 2017

Accepted: 14 June 2018

Published: 16 July 2018

Citation:

Ko A, Jung DE, Kim SH, Kang H-C, Lee JS, Lee ST, Choi JR and Kim HD (2018) The Efficacy of Ketogenic Diet

for Specific Genetic Mutation in Developmental and Epileptic Encephalopathy. Front. Neurol. 9:530.

doi: 10.3389/fneur.2018.00530

\section{The Efficacy of Ketogenic Diet for Specific Genetic Mutation in Developmental and Epileptic Encephalopathy}

\author{
Ara Ko ${ }^{1}$, Da E. Jung ${ }^{2 * t}$, Se H. Kim ${ }^{1}$, Hoon-Chul Kang ${ }^{1}$, Joon S. Lee ${ }^{1}$, Seung T. Lee ${ }^{3}$, \\ Jong R. Choi ${ }^{3}$ and Heung D. Kim ${ }^{1 * t}$
}

${ }^{1}$ Division of Pediatric Neurology, Department of Pediatrics, Epilepsy Research Institute, Severance Children's Hospital, Yonsel University College of Medicine, Seoul, South Korea, ${ }^{2}$ Department of Pediatrics, Ajou University School of Medicine, Suwon, South Korea, ${ }^{3}$ Department of Laboratory Medicine, Severance Hospital, Yonsei University College of Medicine, Seoul, South Korea

Objectives: Pathogenic mutations in developmental and epileptic encephalopathy (DEE) are increasingly being discovered. However, little has been known about effective targeted treatments for this rare disorder. Here, we assessed the efficacy of ketogenic diet (KD) according to the genes responsible for DEE.

Methods: We retrospectively evaluated the data from 333 patients who underwent a targeted next-generation sequencing panel for DEE, 155 of whom had tried KD. Patients showing $\geq 90 \%$ seizure reduction from baseline were considered responders. The KD efficacy was examined at 3, 6, and 12 months after initiation. Patients were divided into those with an identified pathogenic mutation $(n=73)$ and those without $(n=82)$. The $\mathrm{KD}$ efficacy in patients with each identified pathogenic mutation was compared with that in patients without identified genetic mutations.

Results: The responder rate to KD in the patients with identified pathogenic mutations ( $n=73$ ) was $52.1,49.3$, and $43.8 \%$ at 3,6 , and 12 months after initiation, respectively. Patients with mutations in SCN1A ( $n=18$, responder rate $=77.8 \%, p=0.001), K C N Q 2$ $(n=6$, responder rate $=83.3 \%, p=0.022)$, STXBP1 $(n=4$, responder rate $=100.0 \%$, $p=0.015)$, and SCN2A $(n=3$, responder rate $=100.0 \%, p=0.041)$ showed significantly better responses to KD than patients without identified genetic mutations. Patients with CDKL5 encephalopathy $(n=10$, responder rate $=0.0 \%, p=0.031$ ) showed significantly less-favorable responses to KD.

Conclusions: The responder rate to KD remained consistent after KD in DEE patients with specific pathogenic mutations. KD is effective in patients with DEE with genetic etiology, especially in patients with SCN1A, KCNQ2, STXBP1, and SCN2A mutations, but is less effective in patients with $C D K L 5$ mutations. Therefore, identifying the causative gene can help predict the efficacy of KD in patients with DEE.

Keywords: developmental and epileptic encephalopathy, ketogenic diet, next-generation sequencing, mutation, precision medicine 


\section{INTRODUCTION}

Epileptic encephalopathy refers to a group of epileptic disorders that epileptic activities per se cause adverse impacts to the patient's development (1). Developmental and epileptic encephalopathy (DEE) is a recently introduced concept with the advances in genetic diagnosis, as genetic etiologies can cause developmental delay irrespective of epileptic activities (2). The causes of DEE can be various including structural and metabolic etiologies. Moreover, owing to advances in genetic testing technologies, such as next-generation sequencing (NGS), research has revealed that diverse genetic mutations, especially de novo monogenic mutations, constitute a significant portion of the etiologies of DEE (3). The discovery of causative genes for DEE has amplified the efforts to improve our understanding of the pathophysiology of each genetic mutation, with the ultimate objective of precision medicine. For example, ketogenic diet (KD), mechanistic target of rapamycin (mTOR)-inhibitors, and retigabine are effective targeted treatments for glucose transporter type 1 deficiency, mTORopathies, and KCNQ2 encephalopathy, respectively (4-6).

The KD is an effective therapeutic option for various DEE syndromes (7-10), and may be an alternative to pharmacologic therapies. However, the effects of $\mathrm{KD}$ vary among patients and implementing $\mathrm{KD}$ even as a short-term trial is a great challenge for both patients and caregivers, owing to the restrictive diet regimen and potential side effects (11).

Therefore, identifying those patients who are the most likely to respond to KD would be beneficial for determining with whom and when $\mathrm{KD}$ should be initiated. In the present study, we aimed to establish whether the effects of KD differ according to the type of genetic mutation, as confirmed by the targeted NGS gene panel for DEE.

\section{MATERIALS AND METHODS}

\section{Patients}

We retrospectively evaluated the data from patients who were diagnosed with DEE at Severance Children's Hospital, Seoul, South Korea. Inclusion criteria were as follows: (1) patients with epilepsy and cognitive and behavioral impairments who were diagnosed with DEE; (2) patients whose seizures or developmental delays were noticed before the age of 3 years; (3) patients who underwent a targeted NGS gene panel for DEE between January 2016 and March 2017; (4) patients who failed to achieve seizure freedom with adequate trials of two or more antiepileptic drugs (AEDs); and (5) patients who started KD therapy between January 2006 and June 2016. Exclusion criteria were as follows: (1) patients with proven etiologies other than genetic etiology, such as structural, infectious, or immune encephalopathies; and (2) patients who underwent KD therapy for cognitive benefits rather than seizure control, owing to difficulties in assessing the efficacy of $\mathrm{KD}$ by seizure frequency.

Abbreviations: AED, antiepileptic drug; DEE, developmental and epileptic encephalopathy; KD, ketogenic diet; NGS, next-generation sequencing.
This study was approved by the Institutional Review Board of Severance Hospital (IRB No. 4-2017-0699) and written informed consent was waived.

\section{Assessment of Outcomes}

The seizure frequencies before KD (baseline) and at 3, 6, and 12 months after $\mathrm{KD}$ were obtained. The seizure frequency 2 months before KD initiation was defined as the "baseline." When assessing the seizure frequency, the number of seizures that had occurred during the previous 2 months was counted. Patients were considered $\mathrm{KD}$ responders if they showed $\geq 90 \%$ seizure reduction from the baseline seizure frequency, while patients who showed $<90 \%$ seizure reduction were considered nonresponders. The long-term treatment response to $\mathrm{KD}$ was defined as a reduction in seizure frequency at both 6 and 12 months compared to baseline.

The variables we evaluated with respect to their possible predictive value for a responder were as follows: age at seizure onset, sex, number of AEDs being taken at the time of $\mathrm{KD}$ initiation, lead time from seizure onset to $\mathrm{KD}$ initiation, the total duration of $\mathrm{KD}$ implementation, epilepsy syndrome, and pathogenic mutation. Regarding the epilepsy syndrome, the syndromes of each patient at the time of KD implementation were selected. To classify the epilepsy syndrome, we used the classifications outlined by the International League Against Epilepsy in their revised terminology and concepts for organization of seizures and epilepsies (1).

\section{Targeted NGS Panel for DEE}

The targeted NGS panel for DEE at Severance Children's Hospital comprises 172 genes that are known to be related to DEE (Table S1). Using whole-blood samples, genomic DNA was extracted from leukocytes with the QIAamp Blood DNA mini kit (Qiagen, Hilden, Germany). After processing, pooled libraries were sequenced using a MiSeq sequencer (Illumina, San Diego, CA, USA) and the MiSeq Reagent Kit v2 (300 cycles). The interpretation of variants followed the 5-tier classification system recommended by the American College of Medical Genetics and Genomics and the Association for Molecular Pathology (12). Variants considered pathogenic or likely pathogenic according to the American College of Medical Genetics and Genomics and the Association for Molecular Pathology classification system were selected as causative mutations for epileptic encephalopathies (12).

\section{KD}

Patients were instructed to follow either a classic 4:1 or 3:1 KD or modified Atkins diet by the attending pediatric epileptologists (13). Patients immediately began the diet regimen without an initial fasting period, and calories were restricted to $75 \%$ of the recommended daily intake. Screening and follow-up examinations were performed according to the protocol reported by Kang et al. (11), and included measurements of serum $\beta$-hydroxybutyrate and 
urine ketone bodies to assess ketosis and adjust the ratios if required.

\section{Statistical Analysis}

Values are expressed as the median with the interquartile range (IQR) or as the number and percentage. Comparisons between two groups were performed using chi-squared tests or Fisher's exact tests for categorical data or using Mann-Whitney $U$-tests for non-parametric and continuous data. Statistical significance was set at $p<0.05$. The Statistical Package for the Social Sciences software (version 23.0; SPSS Inc., Chicago, IL, USA) was used for all analyses.

\section{RESULTS}

\section{Patients and Clinical Characteristics}

A total of 333 patients (median age 7 [IQR: 3-18] months, 189 [56.8\%] boys) who underwent a targeted NGS gene panel for DEE were included in this retrospective cohort study. Among the
333 patients, 125 (37.5\%) patients received a genetic diagnosis. Of the 125 patients with a monogenic mutation, 73 had tried $\mathrm{KD}$, while 82 of the 208 patients without an identified genetic mutation had tried KD (Figure 1). In total, 155 patients (73 with an identified genetic mutation and 82 without) were subjected to the analysis to determine the KD efficacy. Of the 73 patients with identified genetic mutations, 38 (52.1\%) patients responded to $\mathrm{KD}$ at 3 months, $36(49.3 \%)$ responded at 6 months, and $32(43.8 \%)$ responded at 12 months (Figure 1). Patients were on KD for a median duration of 19 months (IQR: 11.042.5, range: 1-143 months). In the group of 73 patients with an identified genetic mutation, $50(68.5 \%)$ patients were still on $\mathrm{KD}$ after 12 months. Thirteen patients discontinued KD due to inefficacy within 3 months, nine patients discontinued KD between 3 and 6 months (six due to inefficacy, one to aspiration pneumonia, one to recurrent acidosis, and one due to noncompliance), and one patient discontinued KD between 6 and 12 months due to noncompliance. Of the 82 patients without an identified genetic mutation, 27 (32.9\%) patients responded

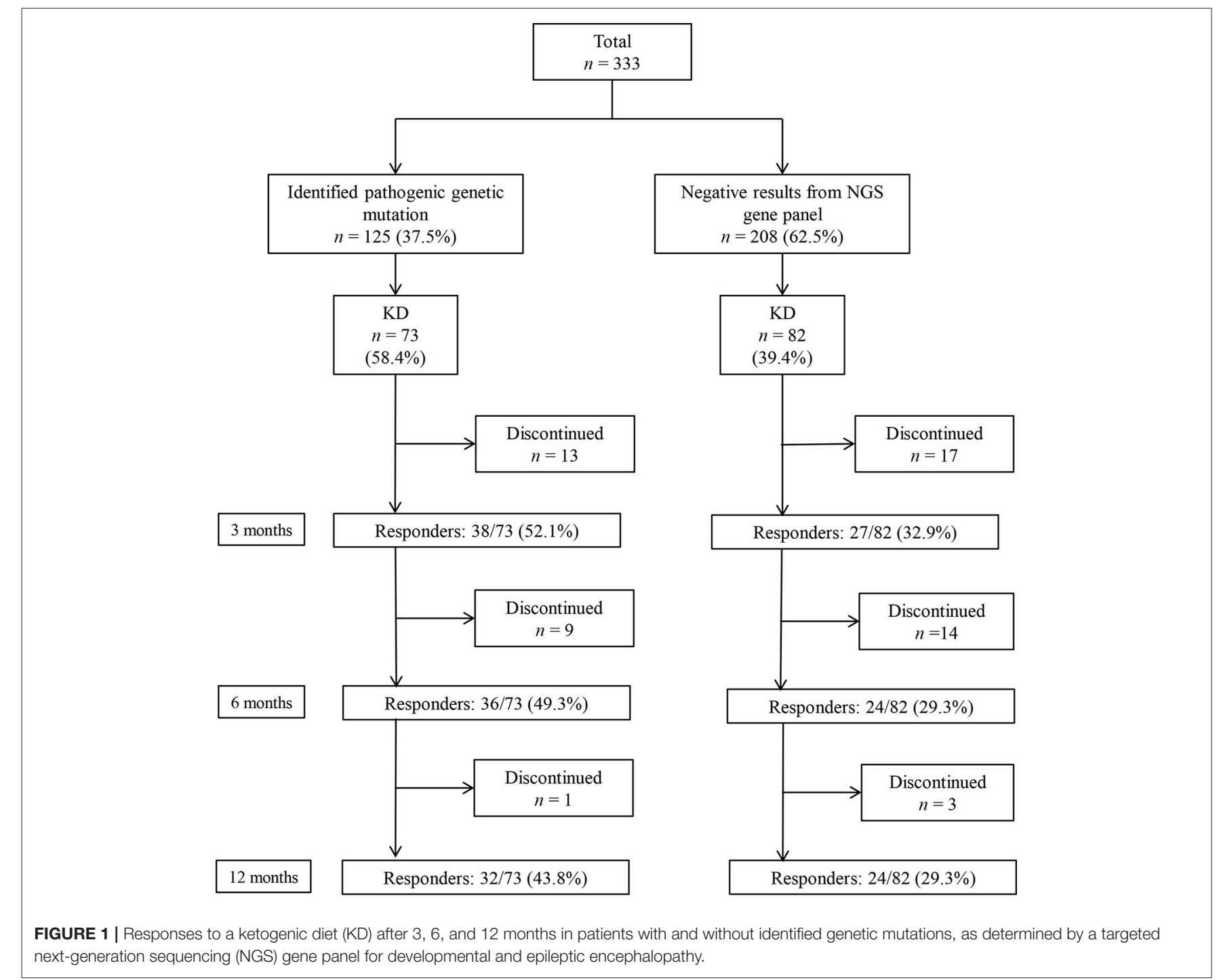


to $\mathrm{KD}$ at 3 months, and $24(29.3 \%)$ responded at both 6 and 12 months. Among the patients without an identified genetic mutation, 48 (58.5\%) patients remained on KD after 12 months. Seventeen patients discontinued the diet within 3 months (eleven due to inefficacy, one to recurrent acidosis, one to recurrent hypoglycemia, one to pancreatitis, one to intolerable diarrhea, and two due to noncompliance). Fourteen patients discontinued the diet between 3 and 6 months (eight due to inefficacy, three to noncompliance, two to aspiration pneumonia, and one due to metabolic encephalopathy), and three patients discontinued the diet between 6 and 12 months (one due to inefficacy and two to noncompliance).

The clinical characteristics of the 155 patients who underwent the NGS gene panel and tried KD are summarized in Table 1. The median age at seizure onset was 6.0 (IQR: 3.0-15.0) months, and $65.2 \%$ of the patients were boys. The median seizure frequency was 7.0 (IQR: 2.0-20.0) seizures per day, while the median number of AEDs patients were taking at the time of KD initiation was 3 (IQR: 3-4). The median lead time from seizure onset to KD initiation was 11.0 (IQR: 5.0-30.5) months. Initially, $24(15.1 \%)$ patients received the $4: 1 \mathrm{KD}, 65(41.9 \%)$ received the $3: 1 \mathrm{KD}$ and $66(42.6 \%)$ received the modified Atkins diet. Regarding the syndromic diagnosis at the time of $\mathrm{KD}$ implementation, West syndrome was the most frequent (67/155 patients, $43.2 \%)$, followed by Lennox-Gastaut syndrome (31/155 patients, 20.0\%), and Dravet syndrome (18/155 patients, $11.6 \%)$, among others. None of the patients underwent epilepsy surgery or the implantation of a vagus nerve stimulation device during the first year of KD (Table 1).

\section{Clinical Characteristics of KD Responders vs. Non-responders Among Patients With DEE}

Among the 155 patients, $65(41.9 \%)$ patients showed $\geq 90 \%$ seizure reduction from baseline at 3 months after $\mathrm{KD}$ initiation and these patients were considered responders. The other $90(58.1 \%)$ patients who showed $<90 \%$ seizure reduction were considered non-responders. No statistically significant differences in the clinical variables including the age at seizure onset, sex, baseline seizure frequency, number of AEDs being taken at the time of $\mathrm{KD}$ implementation, lead time from seizure onset to $\mathrm{KD}$, and $\mathrm{KD}$ ratio, were identified between responders and non-responders (Table 1). However, a significant difference was found between $\mathrm{KD}$ responders and nonresponders regarding the syndromic diagnosis $(p=0.005)$, and significantly more patients with identified pathogenic mutations belonged to the responder group than to the non-responder group ( $p=0.016)$, i.e., patients with an identified genetic mutation responded better to KD.

\section{Clinical Variables of Patients With Identified Mutations vs. Without Identified Mutations by Gene Panel Analysis}

Among the 155 patients, gene panel analysis for DEE showed identified mutations in $73(47.1 \%)$ patients. Clinical variables such as age at seizure onset, baseline seizure frequency before $\mathrm{KD}$, number of AEDs taken before $\mathrm{KD}$, lead time from seizure onset to $\mathrm{KD}$, and $\mathrm{KD}$ ratio were

TABLE 1 | Demographics of patients and comparison between responders and non-responders at 3 months after KD Initiation.

\begin{tabular}{|c|c|c|c|c|}
\hline Clinical variables & Total $(n=155)$ & $\begin{array}{l}\text { Responders* } \\
\quad(n=65)\end{array}$ & $\begin{array}{l}\text { Non-responders } \\
(n=90)\end{array}$ & $p$ \\
\hline Age at seizure onset, months & $6.0(3.0-15.0)$ & $6.0(3.5-11.5)$ & $6.0(3.0-30.8)$ & 0.291 \\
\hline Sex, male & $101(65.2 \%)$ & $38(58.5 \%)$ & $62(68.9 \%)$ & 0.252 \\
\hline Baseline seizure frequency before KD, number per day & $7.0(2.0-20.0)$ & $6.0(1.0-25.0)$ & $10.0(3.0-20.0)$ & 0.435 \\
\hline Number of AEDs before KD & $3(3-4)$ & $3(2-4)$ & $3(3-4)$ & 0.093 \\
\hline Lead time from seizure onset to KD, months & $11.0(5.0-30.0)$ & $13.0(6.0-42.5)$ & $9.5(5.0-24.0)$ & 0.207 \\
\hline KD ratio & & & & 0.113 \\
\hline $4: 1$ & 24 & 8 (33.3\%) & $16(66.7 \%)$ & \\
\hline 3:1 & 65 & $23(35.4 \%)$ & $42(64.6 \%)$ & \\
\hline MAD & 66 & $34(51.5 \%)$ & $32(48.5 \%)$ & \\
\hline Syndromic diagnosis & & & & 0.005 \\
\hline EMAS & 5 & $1(20.0 \%)$ & $4(80.0 \%)$ & \\
\hline Dravet syndrome & 18 & $14(77.8 \%)$ & $4(22.2 \%)$ & \\
\hline Unspecified focal epilepsy & 8 & $2(25.0 \%)$ & $6(75.0 \%)$ & \\
\hline Unspecified generalized epilepsy & 2 & $1(50.0 \%)$ & $1(50.0 \%)$ & \\
\hline West syndrome & 67 & $24(35.8 \%)$ & $43(64.2 \%)$ & \\
\hline Lennox-Gastaut syndrome & 31 & 9 (29.0\%) & $22(71.0 \%)$ & \\
\hline EIMFS & 6 & 2 (33.3\%) & $4(66.7 \%)$ & \\
\hline Landau-Kleffner syndrome & 1 & $1(100.0 \%)$ & $0(0.0 \%)$ & \\
\hline Ohtahara syndrome & 17 & $11(64.7 \%)$ & $6(35.3 \%)$ & \\
\hline Identified pathogenic variant & $73(47.1 \%)$ & $38(58.5 \%)$ & 35 (38.9\%) & 0.016 \\
\hline
\end{tabular}

Data are presented as the median (interquartile range) or as the number (percent).

${ }^{*}$ Responders to $K D$ represent patients who showed $\geq 90 \%$ seizure reduction from baseline.

KD, ketogenic diet; AED, antiepileptic drug; MAD, modified Atkins diet; EMAS, epilepsy with myoclonic atonic seizures; EIMFS, epilepsy of infancy with migrating focal seizures. 
not significantly different between patients with identified mutations and patients without identified mutations (Table 2). Proportion of girls was significantly higher in patients with identified mutations. Also, proportions of patients with identified mutations were significantly different among syndromes.

\section{Causative Monogenic Mutations}

Twenty-five different causative monogenic mutations were found in 73 patients (Table 2). The most common mutations were in the SCN1A gene $(n=18$ [24.7\%]; 10 boys and 8 girls; 11 missense mutations, four nonsense mutations, one splicingsite mutation, one exon 20 deletion, and one whole-gene deletion). Followed by the CDKL5 ( $n=10$ [13.7\%]; 2 boys and 8 girls; four missense mutations, three nonsense mutations, and three splicing-site mutations), KCNQ2 ( $n=6$ [8.2\%]; 4 boys and 2 girls; all missense mutations), and STXBP1 ( $n=4$ [5.5\%]; 2 boys and 2 girls; three missense mutations and one nonsense mutation) genes. Three patients $(4.1 \%)$ had pathogenic mutations in CHD2 (2 boys and 1 girl; two nonsense mutations and one exon 5 deletion), KCNT1 (2 boys and 1 girl; all missense mutations), MECP2 (3 boys; all duplications), SCN2A (1 boy and 2 girls; all missense mutations), and SCN8A (2 boys and 1 girl; all missense mutations), respectively. Other causative monogenic mutations are shown in Table 3.

\section{KD Efficacy According to Causative Monogenic Mutation}

The responder rates of patients with each pathogenic gene were compared with the responder rates of patients without identified genetic mutations at 3, 6, and 12 months after $\mathrm{KD}$ initiation (Table 3). At 3 months after KD implementation, patients with mutations in KCNQ2 $(n=6$, responder rate $=83.3 \%, p=0.022)$, SCN1A $(n=18$, responder rate $=77.8 \%, p=0.001), S C N 2 A$ $(n=3$, responder rate $=100.0 \%, p=0.041)$, and STXBP1 $(n=4$, responder rate $=100.0 \%, p=0.015)$ exhibited significantly better responses to $\mathrm{KD}$ than did patients without identified genetic mutations. In contrast, patients with mutations in CDKL5 $(n=10$, responder rate $=0.0 \%, p=0.031)$ showed significantly poorer responses to $\mathrm{KD}$ than did patients with $\mathrm{DEE}$ without identified genetic mutations. At both 6 and 12 months after KD initiation, patients with mutations in KCNQ2 $(n=6$, responder rate $=83.3 \%, p=0.014$ and $p=0.014)$, SCN1A $(n=18$, responder rate $=77.8$ and $77.8 \%, p<0.001$ and $p=0.014$ ), SCN2A $(n=3$, responder rate $=100.0$ and $100.0 \%, p=0.030$ and $p=0.030)$, and STXBP1 $(n=4$, responder rate $=100.0$ and $100.0 \%, p=0.010$ and $p=0.010$ ) still showed significantly better responses to $\mathrm{KD}$ compared to patients without identified genetic mutations. The responder rates to $\mathrm{KD}$ remained relatively consistent at 3, 6, and 12 months in all patients with pathogenic mutations (52.1, 49.3, and 43.8\%) and patients with each genetic mutation, especially in KCNQ2, SCN1A, SCN2A, and STXBP1 (Figure 2).

TABLE 2 | Demographics of patients and comparison between patient with identified mutations and without identified mutations by gene panel analysis at 3 months after KD Initiation.

\begin{tabular}{|c|c|c|c|c|}
\hline Clinical variables & Total $(n=155)$ & $\begin{array}{l}\text { Identified mutations } \\
\qquad(n=73)\end{array}$ & $\begin{array}{l}\text { Without identified mutations } \\
\qquad(n=82)\end{array}$ & $p$ \\
\hline Age at seizure onset, months & $6.0(3.0-15.0)$ & $5.0(2.0-10.0)$ & $7.0(3.0-17.3)$ & 0.573 \\
\hline Sex, male & $101(65.2 \%)$ & $40(54.8 \%)$ & $61(74.4 \%)$ & 0.012 \\
\hline Baseline seizure frequency before $\mathrm{KD}$, number per day & $7.0(2.0-20.0)$ & $6.0(1.8-200)$ & $7.0(2.0-35.0)$ & 0.675 \\
\hline Number of AEDs before KD & $3(3-4)$ & $3(2-4)$ & $3(3-4)$ & 0.254 \\
\hline Lead time from seizure onset to KD, months & $11.0(5.0-30.0)$ & $13.0(5.0-37.0)$ & $10.0(5.0-24.0)$ & 0.291 \\
\hline KD ratio & & & & 0.249 \\
\hline $4: 1$ & 24 & $9(37.5 \%)$ & $15(62.5 \%)$ & \\
\hline $3: 1$ & 65 & $28(43.1 \%)$ & 37 (56.9\%) & \\
\hline MAD & 66 & $36(54.5 \%)$ & $30(45.5 \%)$ & \\
\hline Syndromic diagnosis & & & & $<0.001$ \\
\hline EMAS & 5 & $2(40.0 \%)$ & $3(60.0 \%)$ & \\
\hline Dravet syndrome & 18 & 18 (100.0\%) & $0(0.0 \%)$ & \\
\hline Unspecified focal epilepsy & 8 & $1(12.5 \%)$ & 7 (87.5\%) & \\
\hline Unspecified generalized epilepsy & 2 & 2 (100.0\%) & $0(0.0 \%)$ & \\
\hline West syndrome & 67 & 20 (29.9\%) & 47 (70.1\%) & \\
\hline Lennox-Gastaut syndrome & 31 & $12(38.7 \%)$ & $19(61.3 \%)$ & \\
\hline EIMFS & 6 & $3(50.0 \%)$ & $3(50.0 \%)$ & \\
\hline Landau-Kleffner syndrome & 1 & $0(0.0 \%)$ & $1(100.0 \%)$ & \\
\hline Ohtahara syndrome & 17 & $15(88.2 \%)$ & $2(11.8 \%)$ & \\
\hline
\end{tabular}

Data are presented as the median (interquartile range) or as the number (percent).

KD, ketogenic diet; AED, antiepileptic drug; MAD, modified Atkins diet; EMAS, epilepsy with myoclonic atonic seizures; EIMFS, epilepsy of infancy with migrating focal seizures. 
TABLE 3 | Responder rates to ketogenic diet according to the identified pathogenic gene, with the $P$ value for the comparison with the responder rate of patients without identified genetic mutations.

\begin{tabular}{|c|c|c|c|c|c|c|}
\hline \multirow[t]{2}{*}{ Pathogenic gene $(n=73)$} & \multicolumn{2}{|c|}{ At 3 months } & \multicolumn{2}{|c|}{ At 6 months } & \multicolumn{2}{|c|}{ At 12 months } \\
\hline & Responders & $p$ & Responders & $p$ & Responders & $p$ \\
\hline$A R X(1)$ & $1 / 1(100.0 \%)$ & 0.337 & 1/1 (100.0\%) & 0.301 & $1 / 1(100.0 \%)$ & 0.301 \\
\hline CACNA1A (1) & 1/1 (100.0\%) & 0.337 & 0/1 (0.0\%) & 1.000 & 0/1 (0.0\%) & 1.000 \\
\hline CDKL5 (10) & 0/10 (0.0\%) & 0.031 & 0/10 (0.0\%) & 0.058 & 0/10 (10.0\%) & 0.058 \\
\hline CHD2 (3) & 1/3 (33.3\%) & 1.000 & 0/3 (0.0\%) & 0.555 & 0/3 (0.0\%) & 0.555 \\
\hline CLCN4 (1) & $0 / 1(0.0 \%)$ & 1.000 & 0/1 (0.0\%) & 1.000 & 0/1 (0.0\%) & 1.000 \\
\hline COL4A1 (2) & $1 / 2(50.0 \%)$ & 1.000 & $1 / 2$ (50.0\%) & 0.509 & $1 / 2(50.0 \%)$ & 0.509 \\
\hline EEF1A2 (2) & 0/2 (0.0\%) & 1.000 & 0/2 (0.0\%) & 1.000 & 0/2 (0.0\%) & 1.000 \\
\hline GNAO1 (1) & 0/1 (0.0\%) & 1.000 & 0/1 (0.0\%) & 1.000 & 0/1 (0.0\%) & 1.000 \\
\hline IQSEC2 (1) & 0/1 (0.0\%) & 1.000 & 0/1 (0.0\%) & 1.000 & 0/1 (0.0\%) & 1.000 \\
\hline KANSL1 (1) & 1/1 (100.0\%) & 0.337 & 1/1 (100.0\%) & 0.301 & 1/1 (100.0\%) & 0.301 \\
\hline KCNA1 (1) & 0/1 (0.0\%) & 1.000 & 0/1 (0.0\%) & 1.000 & 0/1 (0.0\%) & 1.000 \\
\hline KCNB1 (2) & 2/2 (100.0\%) & 0.116 & 2/2 (100.0\%) & 0.093 & 2/2 (100.0\%) & 0.093 \\
\hline KCNQ2 (6) & 5/6 (83.3\%) & 0.022 & 5/6 (83.3\%) & 0.014 & 5/6 (83.3\%) & 0.014 \\
\hline KCNT1 (3) & 0/3 (0.0\%) & 0.548 & 0/3 (0.0\%) & 0.555 & 0/3 (33.3\%) & 0.555 \\
\hline MECP2 (3) & 0/3 (0.0\%) & 0.548 & 0/3 (0.0\%) & 0.555 & 0/3 (0.0\%) & 0.555 \\
\hline SCN1A (18) & 14/18 (77.8\%) & 0.001 & 14/18 (77.8\%) & $<0.001$ & $11 / 18(61.1 \%)$ & 0.014 \\
\hline SCN2A (3) & $3 / 3$ (100\%) & 0.041 & 3/3 (100.0\%) & 0.030 & $3 / 3$ (100.0\%) & 0.030 \\
\hline SCN3A (1) & 1/1 (100.0\%) & 0.337 & 1/1 (100.0\%) & 0.301 & 1/1 (100.0\%) & 0.301 \\
\hline SCN8A (3) & 0/3 (0.0\%) & 0.548 & 0/3 (0.0\%) & 0.555 & 0/3 (0.0\%) & 0.555 \\
\hline SLC6A1 (1) & $1 / 1(100.0 \%)$ & 0.337 & 1/1 (100.0\%) & 0.301 & 1/1 (100.0\%) & 0.301 \\
\hline SLC9A6 (2) & 1/2 (50.0\%) & 1.000 & 1/2 (50.0\%) & 0.509 & 1/2 (50.0\%) & 0.509 \\
\hline STXBP1 (4) & 4/4 (100\%) & 0.015 & 4/4 (100\%) & 0.010 & 4/4 (100.0\%) & 0.010 \\
\hline SYNGAP (1) & 0/1 (0.0\%) & 1.000 & 0/1 (0.0\%) & 1.000 & 0/1 (0.0\%) & 1.000 \\
\hline WWOX (1) & $1 / 1(100.0 \%)$ & 0.337 & 1/1 (100.0\%) & 0.301 & 1/1 (100.0\%) & 0.301 \\
\hline ZEB2 (1) & $1 / 1(100.0 \%)$ & 0.337 & 1/1 (100.0\%) & 0.301 & 0/1 (0.0\%) & 1.000 \\
\hline
\end{tabular}

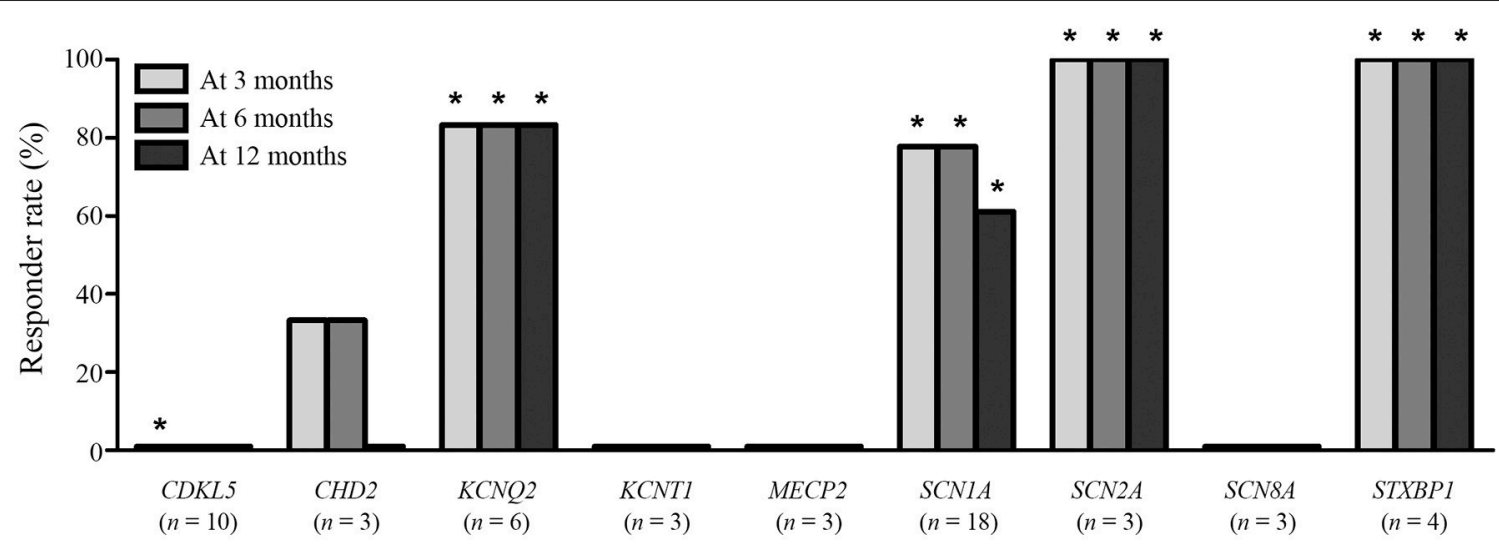

FIGURE 2 | Responses to a ketogenic diet after 3, 6, and 12 months according to the identified pathogenic genetic mutations found in $\geq 3$ patients. *Responder rates that are significantly lower (CDKL5) or higher (the others) than the responder rate of patients without an identified genetic mutation.

\section{KD Efficacy According to Causative Mutations in Epilepsy Syndromes}

As mentioned above, when patients were divided by syndromic diagnoses at the time of $\mathrm{KD}$ initiation, West syndrome was the most frequent, followed by Lennox-Gastaut syndrome, Dravet syndrome, and Ohtahara syndrome (Table 1). Among 67 West syndrome patients, pathogenic mutations were found in 20 (29.9\%) patients, including 9 patients with CDKL5 mutations. Mutations in other genes were found in $\leq 2$ patients for each gene. Responder rate of West syndrome patients with 
CDKL5 mutations was significantly lower than responder rate of West syndrome patients without CDKL5 mutations (0.0 vs. $41.4 \%, p=0.021)$. Among 31 Lennox-Gastaut syndrome patients, $12(38.7 \%)$ patients were identified with pathogenic mutations. Causative genes with mutations in Lennox-Gastaut syndrome patients were heterogeneous to analyze, with 3 boys with mutations in MECP2 gene and other 9 patients all identified with different genes from each other. All 18 Dravet syndrome patients were identified with mutations in SCN1A gene, and $14(77.8 \%)$ patients showed a good response to $\mathrm{KD}$ at 3 months since initiation of $\mathrm{KD}$. Of 17 Ohtahara syndrome patients, $15(88.2 \%)$ patients were identified with causative mutations, including 6 KCNQ2 mutations, 3 STXBP1 mutations, and other mutations found in single patient each. Responder rate of Ohtahara syndrome patients with KCNQ2 or STXBP1 mutations showed a significantly better response to $\mathrm{KD}$ after 3 months compared to Ohtahara syndrome patients without KCNQ2 or STXBP1 mutations (88.9 vs. $37.5 \%$, $p=0.043)$.

\section{DISCUSSION}

In our study, among the patients with DEE with various pathogenic mutations, patients with $S C N 2 A, S T X B P 1, K C N Q 2$, and SCN1A mutations in particular showed better responses to $\mathrm{KD}$, with responder rates of $100,100,83.3$, and $77.8 \%$ respectively. However, patients with CDKL5 mutations sho wed poorer responses to $\mathrm{KD}$ (responder rate $=0.0 \%$ ) at 3 months after implementation. The responder rate to $\mathrm{KD}$ remained consistent at 3, 6, and 12 months after diet initiation when examined according to genotype.

The monogenic mutations that are responsible for DEE are increasingly being discovered with the advent of the NGS technique and targeted NGS or gene panel studies have been suggested as the most cost-effective genetic testing method for patients with DEE (14). Of the 333 patients in our study who underwent a DEE gene panel, 125 (37.5\%) patients had an identified causative monogenic mutation; this incidence rate is similar to those reported in previous studies $(15,16)$, and such findings collectively support the usefulness of NGS panels in searching for the genetic causes of DEE. Moreover, our data provide useful insights regarding whom and when to recommend KD therapy for patients with gene-associated DEE.

To date, few reports on the efficacy of KD in patients with DEE with specific genotypes have been published (17-19), and of these, most are anecdotal reports or include short-term evaluations of the efficacy in only one specific genotype. In the present study, we assessed the long-term response to KD (over 12 months) in patients with each genotype of DEE. Additionally, we focused on patients who showed definite improvements after KD by strictly regarding only those patients who showed $\geq 90 \%$ seizure reduction as responders. Therefore, this study is significant because it is the first to assess the long-term efficacy of $\mathrm{KD}$ according to the various genetic causes of DEE. Moreover, we revealed the genotypes that are the most likely to show significant seizure reductions after $\mathrm{KD}$.
The KD is reportedly effective for patients with DEE syndromes such as Dravet syndrome, West syndrome, LennoxGastaut syndrome, and epilepsy with myoclonic atonic seizures (7-10, 20-22). Efforts to identify phenotypes with clinical variables that can help predict which patients will show a better response to $\mathrm{KD}$ have been made (23). Our study showed that children with specific syndromes, especially those with an identified genetic cause, responded better to $\mathrm{KD}$ at 3 months than did those with syndromes without identified genetic causes. However, none of the other clinical variables we evaluated were related to a good $\mathrm{KD}$ response. In addition to the type of epileptic syndrome, we compared the efficacy of KD between different genotypes in order to reveal which genotypes are the most likely to respond well to KD. Our analysis demonstrated that patients with SCN2A, STXBP1, KCNQ2, and SCN1A mutations had more favorable responses to $\mathrm{KD}$ than did those without genetic mutations.

Previous studies have shown that patients with Dravet syndrome with SCN1A mutations respond well to $\operatorname{KD}(21,24)$, as did a patient with an $S C N 2 A$ mutation who was treated with a modified Atkins diet (19). Here, as well as in several other studies $(25,26)$, patients with CDKL5 mutations showed poor responses to or only short-term efficacy of KD. Therefore, our findings regarding these genes are consistent with those of previous studies and confirm that patients with epileptic encephalopathy with SCN1A and SCN2A mutations respond well to $\mathrm{KD}$, while patients with CDKL5 mutations respond poorly to $\mathrm{KD}$. Although prior studies showed that for patients with STXBP1 encephalopathy, the response to KD was either slight or none (two case reports, each with one patient) $(27,28)$, in our study, all four patients with STXBP1 mutations responded well to KD.

Among patients with West syndrome, patients with CDKL5 mutations showed a significantly poorer response to KD than other West syndrome patients without CDKL5 mutation. Among patients with Ohtahara syndrome, patients with KCNQ2 or STXBP1 mutations showed a significantly better response to $\mathrm{KD}$ than the others in this study. This is in line with the results from analysis by causative mutations in all DEE, showing that causative mutation is the important determining factor of response to $\mathrm{KD}$ as much as syndromic diagnoses in DEE. However, further study with larger sample size is warranted.

To our knowledge, no reports have evaluated the efficacy of $\mathrm{KD}$ in patients with KCNQ2-related neonatal epileptic encephalopathy. Here, $83.3 \%$ of patients with KCNQ2 (5/6 patients) mutations responded to $\mathrm{KD}$. However, none of our patients with CHD2 (0/3), KCNT1 (0/3), SCN8A (0/3), and $\operatorname{MECP} 2(0 / 3$, all boys) mutations showed a response to $\mathrm{KD}$. Unfortunately, in patients with these genetic mutations, the KD efficacy was not statistically significant than was that in patients without identified genetic mutations owing to the small number of patients with each genetic mutation.

We understand the limitations of using an observational approach in this study and acknowledge that prospective or randomized controlled studies can provide stronger evidence regarding the efficacy of $\mathrm{KD}$ in patients with $\mathrm{DEE}$ with each pathogenic variant. We also acknowledge the very small sample 
size of this study, due to rare nature of DEE with identified pathogenic mutations. This study should therefore be regarded as elementary results demonstrating that specific genetic mutations show different response to KD. Due to the retrospective study design, the effects of $\mathrm{KD}$ on the developmental outcomes of these children were not evaluated. For patients without identified genetic mutations, some proportion of them is suspected to have a genetic origin owing to unknown pathogenic genes or genes that were not included in our NGS panel. Despite its limitations, the present study provides the first overview of the efficacy of KD in patients with DEE according to genotype and may serve as a basis for administering precision medicine.

\section{CONCLUSIONS}

The KD was particularly effective in patients with DEE caused by SCN2A, STXBP1, KCNQ2, and SCN1A mutations and was not effective in patients with DEE caused by CDKL5 mutations. These results will provide a rational basis for considering early, targeted KD treatment rather than the traditional trial and error approach to epilepsy therapy in this group of patients. Furthermore, our

\section{REFERENCES}

1. Berg AT, Berkovic SF, Brodie MJ, Buchhalter J, Cross JH, van Emde Boas W, et al. Revised terminology and concepts for organization of seizures and epilepsies: report of the ILAE Commission on Classification and Terminology, 2005-2009. Epilepsia (2010) 51:676-85. doi: 10.1111/j.1528-1167.2010.02522.x

2. Scheffer IE, Berkovic S, Capovilla G, Connolly MB, French J, Guilhoto L, et al. ILAE classification of the epilepsies: position paper of the ILAE Commission for Classification and Terminology. Epilepsia (2017) 58:512-21. doi: 10.1111/epi.13709

3. Moller RS, Dahl HA, Helbig I. The contribution of next generation sequencing to epilepsy genetics. Expert Rev Mol Diagn. (2015) 15:1531-8. doi: 10.1586/14737159.2015.1113132

4. Kass HR, Winesett SP, Bessone SK, Turner Z, Kossoff EH. Use of dietary therapies amongst patients with GLUT1 deficiency syndrome. Seizure (2016) 35:83-7. doi: 10.1016/j.seizure.2016.01.011

5. Krueger DA, Wilfong AA, Holland-Bouley K, Anderson AE, Agricola K, Tudor C, et al. Everolimus treatment of refractory epilepsy in tuberous sclerosis complex. Ann Neurol. (2013) 74:679-87. doi: 10.1002/ana.23960

6. Gunthorpe MJ, Large CH, Sankar R. The mechanism of action of retigabine (ezogabine), a first-in-class $\mathrm{K}+$ channel opener for the treatment of epilepsy. Epilepsia (2012) 53:412-24. doi: 10.1111/j.1528-1167.2011.03365.x

7. Caraballo RH, Cersosimo RO, Sakr D, Cresta A, Escobal N, Fejerman N, et al. Ketogenic diet in patients with Dravet syndrome. Epilepsia (2005) 46:1539-44. doi: 10.1111/j.1528-1167.2005.05705.x

8. Eun SH, Kang HC, Kim DW, Kim HD. Ketogenic diet for treatment of infantile spasms. Brain Dev. (2006) 28:566-71. doi: 10.1016/j.braindev.2006.03.011

9. Lemmon ME, Terao NN, Ng YT, Reisig W, Rubenstein JE, Kossoff EH, et al. Efficacy of the ketogenic diet in Lennox-Gastaut syndrome: a retrospective review of one institution's experience and summary of the literature. Dev Med Child Neurol. (2012) 54:464-8. doi: 10.1111/j.1469-8749.2012.04233.x

10. Wiemer-Kruel A, Haberlandt E, Hartmann H, Wohlrab G, Bast T. Modified Atkins diet is an effective treatment for children with Doose syndrome. Epilepsia (2017) 58:657-62. doi: 10.1111/epi.13701

11. Kang HC, Chung DE, Kim DW, Kim HD. Early- and late-onset complications of the ketogenic diet for intractable epilepsy. Epilepsia (2004) 45:1116-23. doi: 10.1111/j.0013-9580.2004.10004.x data may help avoid overtreatment with $\mathrm{KD}$ in patients with $\mathrm{DEE}$ with CDKL5 mutations.

\section{AUTHOR CONTRIBUTIONS}

DJ and HK conceptualized and designed the study, reviewed and revised the manuscript. DJ and AK carried out the initial analyses, drafted the initial manuscript. SL and JC carried out the initial analyses. SK, H-CK, and JL collected data and critically reviewed the manuscript.

\section{FUNDING}

This study was supported by faculty research grant of Yonsei University College of Medicine for 2011 (6-2011-0079).

\section{SUPPLEMENTARY MATERIAL}

The Supplementary Material for this article can be found online at: https://www.frontiersin.org/articles/10.3389/fneur. 2018.00530/full\#supplementary-material
12. Richards S, Aziz N, Bale S, Bick D, Das S, Gastier-Foster J, et al. Standards and guidelines for the interpretation of sequence variants: a joint consensus recommendation of the American College of Medical Genetics and Genomics and the Association for Molecular Pathology. Genet Med. (2015) 17:405-24. doi: 10.1038/gim.2015.30

13. Kossoff EH, McGrogan JR, Bluml RM, Pillas DJ, Rubenstein JE, Vining EP, et al. A modified Atkins diet is effective for the treatment of intractable pediatric epilepsy. Epilepsia (2006) 47:421-4. doi: 10.1111/j.1528-1167.2006.00438.x

14. Gokben S, Onay H, Yilmaz S, Atik T, Serdaroglu G, Tekin H, et al. Targeted next generation sequencing: the diagnostic value in earlyonset epileptic encephalopathy. Acta Neurol Belg. (2017) 117:131-8. doi: 10.1007/s13760-016-0709-z

15. Mercimek-Mahmutoglu S, Patel J, Cordeiro D, Hewson S, Callen D, Donner EJ, et al. Diagnostic yield of genetic testing in epileptic encephalopathy in childhood. Epilepsia (2015) 56:707-16. doi: 10.1111/epi.12954

16. de Kovel CG, Brilstra EH, van Kempen MJ, Van't Slot R, Nijman IJ, Afawi Z, et al. Targeted sequencing of 351 candidate genes for epileptic encephalopathy in a large cohort of patients. Mol Genet Genomic Med. (2016) 4:568-80. doi: $10.1002 / \mathrm{mgg} 3.235$

17. Mori T, Imai K, Oboshi T, Fujiwara Y, Takeshita S, Saitsu H, et al. Usefulness of ketogenic diet in a girl with migrating partial seizures in infancy. Brain Dev. (2016) 38:601-4. doi: 10.1016/j.braindev.2015.12.012

18. Liebhaber GM, Riemann E, Baumeister FA. Ketogenic diet in Rett syndrome. J Child Neurol. (2003) 18:74-5. doi: 10.1177/08830738030180011801

19. Wong VC, Fung CW, Kwong AK. SCN2A mutation in a Chinese boy with infantile spasm - response to modified Atkins diet. Brain Dev. (2015) 37:72932. doi: $10.1016 /$ j.braindev.2014.10.008

20. Laux L, Blackford R. The ketogenic diet in Dravet syndrome. J Child Neurol. (2013) 28:1041-4. doi: 10.1177/0883073813487599

21. Dressler A, Trimmel-Schwahofer P, Reithofer E, Muhlebner A, Groppel G, Reiter-Fink E, et al. Efficacy and tolerability of the ketogenic diet in Dravet syndrome - comparison with various standard antiepileptic drug regimen. Epilepsy Res. (2015) 109:81-9. doi: 10.1016/j.eplepsyres.2014. 10.014

22. Stenger E, Schaeffer M, Cances C, Motte J, Auvin S, Ville D, et al. Efficacy of a ketogenic diet in resistant myoclono-astatic epilepsy: a French multicenter retrospective study. Epilepsy Res. (2017) 131:64-9. doi: 10.1016/j.eplepsyres.2017.02.005 
23. Schoeler NE, Cross JH, Sander JW, Sisodiya SM. Can we predict a favourable response to ketogenic diet therapies for drug-resistant epilepsy? Epilepsy Res. (2013) 106:1-16. doi: 10.1016/j.eplepsyres.2013.06.002

24. Nabbout R, Copioli C, Chipaux M, Chemaly N, Desguerre I, Dulac O, et al. Ketogenic diet also benefits Dravet syndrome patients receiving stiripentol: a prospective pilot study. Epilepsia (2011) 52:e54-7. doi: 10.1111/j.1528-1167.2011.03107.x

25. Muller A, Helbig I, Jansen C, Bast T, Guerrini R, Jahn J, et al. Retrospective evaluation of low long-term efficacy of antiepileptic drugs and ketogenic diet in 39 patients with CDKL5-related epilepsy. Eur J Paediatr Neurol. (2016) 20:147-51. doi: 10.1016/j.ejpn.2015.09.001

26. Lim Z, Wong K, Olson HE, Bergin AM, Downs J, Leonard H, et al. Use of the ketogenic diet to manage refractory epilepsy in CDKL5 disorder: experience of $>100$ patients. Epilepsia (2017) 58:1415-22. doi: 10.1111/epi.13813

27. Saitsu H, Hoshino H, Kato M, Nishiyama K, Okada I, Yoneda Y, et al. Paternal mosaicism of an STXBP1 mutation in OS. Clin Genet. (2011) 80:484-8. doi: 10.1111/j.1399-0004.2010.01575.x
28. Weckhuysen S, Holmgren P, Hendrickx R, Jansen AC, Hasaerts D, Dielman C, et al. Reduction of seizure frequency after epilepsy surgery in a patient with STXBP1 encephalopathy and clinical description of six novel mutation carriers. Epilepsia (2013) 54:e74-80. doi: 10.1111/epi. 12124

Conflict of Interest Statement: The authors declare that the research was conducted in the absence of any commercial or financial relationships that could be construed as a potential conflict of interest.

Copyright (c) 2018 Ko, Jung, Kim, Kang, Lee, Lee, Choi and Kim. This is an openaccess article distributed under the terms of the Creative Commons Attribution License (CC BY). The use, distribution or reproduction in other forums is permitted, provided the original author(s) and the copyright owner(s) are credited and that the original publication in this journal is cited, in accordance with accepted academic practice. No use, distribution or reproduction is permitted which does not comply with these terms. 